\title{
Diseño, simulación y análisis del proceso de extrusión de un perfil termoplástico en PVC rígido para optimizar el diseño de la matriz y los parámetros
}

\author{
Design, simulation and analysis of extrusion \\ process for thermoplastic rigid PVC profile \\ to optimize the design of the die and \\ the parameters
}

Carlos José Salvador Tomassini ${ }^{1}$

\begin{abstract}
${ }^{1}$ Departamento de Plásticos y Elastómeros, Instituto Politécnico Superior, Universidad Nacional de Rosario, Rosário, Santa Fé, Argentina. Escuela Posgrado y Educación Continua, Facultad de Cs.Ex., Ingeniería y Agrimensura, Universidad Nacional Rosario.

e-mail: ctomassini8@gmail.com
\end{abstract}

\section{RESUMEN}

El objetivo del presente trabajo fue verificar y optimizar el diseño de un cabezal para fabricar un perfil termoplástico en PVC rígido utilizando la simulación del flujo en dicha matriz mediante el uso de Dinámica de Fluidos Computacional (CFD) y por medio de pruebas en extrusión, empleando un calibrador experimental, se obtuvieron la geometría y las dimensiones requeridas del mismo. El material utilizado fue un compuesto de PVC rígido el cual es difícil de procesar por su debilidad intrínseca, ya que se degrada a los $140^{\circ} \mathrm{C}$. Por medio de un reómetro capilar especial se determinaron los datos reológicos $\mathrm{k}$ y n del modelo de Ley de Potencia los cuales se utilizaron como variables de entrada en el software junto a la geometría del canal de flujo en el cabezal. La geometría es dibujada mediante un software de diseño asistido por computadora en tres dimensiones (CAD-3D). El calibrador se diseñó y dibujó en un software CAD-3D y luego se lo construye, montándose en el equipo extrusor. Se realizó la extrusión con los parámetros de proceso: caudal (velocidad giro tornillo) y temperatura del material utilizados en el software de simulación. Se comparó los resultados obtenidos de la extrusión: geometría y dimensiones finales del perfil, caudal másico, presión y temperatura en el cabezal con los obtenidos por la simulación, siendo los mismos satisfactorios. De la simulación se determinó la distancia óptima a la cual las deformaciones del perfil fundido a la salida del cabezal son mínimas y permitieron que el mismo pueda calibrarse a las dimensiones requeridas del perfil. Además se obtuvieron las distribuciones de velocidades, presiones y deformaciones de corte, las cuales dieron una indicación de las propiedades mecánicas del perfil, como ser las tensiones internas y resistencia a esfuerzos en servicio, además de los valores dimensionales y de la geometría final, de manera que se logró determinar que el diseño del cabezal existente no es el correcto.

Palabras claves: simulación CFD, extrusión, PVC(Policloruro de Vinilo), diseño, reología.

\section{ABSTRACT}

The objective of the present work was to verify and optimize the die design to manufacture a thermoplastic profile in rigid PVC using the simulation of the flow in the mentioned matrix through the use of Computational Fluid Dynamics (CFD) and through tests in extrusion, using an experimental calibrator, the required geometry and dimensions were obtained. The material used was a rigid PVC compound which is difficult to process because of its intrinsic weakness, since it degrades to $140^{\circ} \mathrm{C}$. By means of a special capillary rheometer the rheological data $\mathrm{k}$ and $\mathrm{n}$ of the Power Law model were determined, which was used as input variables in the software along with the flow channel geometry in the head. The geometry is drawn using computeraided design software in three dimensions (CAD-3D). The calibrator was designed and drawn in CAD-3D software and then built, mounted on the extruder. Extrusion was performed with the process parameters: flow rate (screw speed) and temperature of the material used in the simulation software. The results of the extrusion were compared: geometry and final dimensions of the profile, mass flow, pressure and temperature in the head with those obtained by the simulation, being the same satisfactory. From the simulation, the opti- 
mum distance at which the deformations of the cast profile at the exit of the die determined was minimal and allowed to be calibrated to the required profile dimensions. In addition, the velocity distributions and shear deformations were obtained, which gave an indication of the dimensional and geometric and mechanical physical properties of resistance to the stresses of the profile, so that it was possible to determine that the design of the existing head is not right.

Key words: simulation CFD, extrusion, PVC (polyvinylchloride), design, rheology.

\section{INTRODUCCIÓN}

La extrusión de un perfil termoplástico es la producción de un producto continuo que en estado fundido plastificado es forzado a pasar por un cabezal que posee la geometría final deseada y en particular los de PVC tienen un uso cada vez más frecuente en la fabricación de marcos para puertas y ventanas y otros productos para la construcción por sus mejores propiedades (aislante térmico y acústico, más liviano, ecológico, etc.) con respecto a los materiales convencionales (acero y aluminio).

Los perfiles de PVC pueden tener secciones transversales de geometría compleja, requerir reducidas tolerancias dimensionales y durante su procesamiento éste compuesto en estado fundido genera un gas corrosivo provocando que el diseño de los cabezales para la producción de éstos perfiles se convierta en una difícil tarea.

Los métodos tradicionales de diseño dependen en gran medida de la experiencia del diseñador y utilizando el método de prueba y error, pudiendo requerirse para el desarrollo de un cabezal complejo de $15 \mathrm{o}$ más de éstos ciclos [1], elevando los costos y tiempos de desarrollo y construcción. Por lo cual el uso de la simulación por computadora, utilizando técnicas numéricas específicas que emplean la ciencia de la Dinámica de Fluidos Computacional (CFD) [2], permite acelerar estos procesos, realizando las consideraciones necesarias previas a su fabricación.

La modelización del diseño utilizando la simulación por computadora se transforma en una herramienta de gran importancia para la industria, en especial para las pequeñas y medianas empresas las cuales no utilizan o tienen acceso a herramientas computacionales y personal capacitado para el diseño de cabezales y la optimización del proceso de extrusión de perfiles termoplásticos en nuestro país.

Para poder simular correctamente es esencial conocer y caracterizar correctamente la compleja reología de éstos compuestos (formados por materiales de distinto comportamiento) y otras importantes propiedades de los mismos como ser su densidad, temperatura de procesamiento, etc. [1].

El objetivo de éste trabajo es mediante el uso de la modelización y simulación por computadora con el uso de la CFD, conocer el comportamiento del flujo del material fundido de un cabezal existente para un perfil en PVC rígido y comparar los resultados con las pruebas en extrusora para mejorar la calidad final de dicho perfil (dimensiones, geometría, evitar o minimizar tensiones internas, deformaciones, etc.); además complementar éste proceso con el diseño óptimo de un calibrador modelizado y fabricado especialmente para el mismo [3].

Mediante la simulación se determinó también la distancia óptima a la cual las deformaciones del perfil fundido a la salida del cabezal son mínimas y permiten que el mismo pudiera calibrarse a las dimensiones finales requeridas dentro del porcentaje de error recomendado [4].

Por último se obtuvieron las distribuciones de velocidades y deformaciones de corte que sufre el flujo del material fundido desde que ingresa al cabezal hasta que se introduce en el calibrador, las cuales nos brindaron una indicación de las propiedades mecánicas (elevadas tensiones internas de cizallamiento que afecta a la resistencia a los esfuerzos que tiene que soportar posteriormente el perfil en servicio) que determinaron, en lo que respecta a éste aspecto, que el diseño del cabezal existente no es el correcto.

\section{MATERIALES Y MÉTODOS}

\subsection{Material}

El material objeto de investigación es un compuesto de PVC rígido de la empresa ALFAVINIL S.A., código: RE97/1-773, el cual posee en su composición diferentes tipos de materiales (resina, plastificante, aditivos, cargas minerales, pigmentos, estabilizantes térmicos y otros.).

Por su composición química es muy sensible térmicamente, degradándose al ser calentado a partir de los $140^{\circ} \mathrm{C}$, por eso, para su procesamiento, además de contener en su formulación un estabilizante térmico, se requiere el uso de extrusores especiales, con tornillos de baja relación de compresión 2:1). 
En la Tabla 1 se detalla la composición del compuesto de PVC rígido que se utilizó para este trabajo. Tabla 1: Composición química del compuesto de PVC rígido de la empresa ALFAVINIL código RE97/1-773.

\begin{tabular}{l|c|l}
\hline \multicolumn{1}{c|}{ MATERIAL } & PORCENTAJE (\%) & \multicolumn{1}{c}{ DETALLES } \\
\hline Contenido de la resina & 66 & Proveedor: Solvay Argentina \\
\hline Contenido de plastificante & 2 & Origen vegetal, utilizado como co-estabilizante \\
\hline Contenido de estabilizante & 2 & $\begin{array}{l}\text { Pack estabilizante-lubricante a base de sulfato } \\
\text { tribásico de Plomo }\end{array}$ \\
\hline Contenido de otros elementos & 24 & $\begin{array}{l}\text { Carbonato de calcio micronizado recubierto, tama- } \\
\text { ño medio partícula 1,6 micrones }\end{array}$ \\
\hline Dióxido de titanio & 6 & Alta opacidad \\
\hline
\end{tabular}

Una forma práctica de identificar un compuesto de PVC es mediante su valor K, el cual es el índice de viscosidad relativa [5] y que en nuestro caso tiene un valor de 65; el mismo se determina mediante un ensayo de una solución con una concentración de 5g/lt. de PVC-U en ciclohexanona, midiendo el tiempo de paso por un capilar que contiene el viscosímetro de Ostwald; éste ensayo también fué realizado por la empresa ALFAVINIL S.A.

La mayoría de los polímeros fundidos se comportan como fluidos no-newtonianos, es decir su viscosidad de corte $(\eta)$, a una temperatura constante, depende de la deformación de corte $(\gamma)$, pudiendo definir la misma (Ecuación 1) como el cociente entre la tensión de corte $(\tau)$ y la deformación de corte $(\gamma)$ [4].

$$
\eta=\tau / \gamma
$$

Los termoplásticos, en especial el PVC, se clasifican como pseudoplásticos, es decir su viscosidad de corte decrece al aumentar la deformación de corte $(\gamma)$.

Para realizar una simulación se debe poseer información precisa del comportamiento del material bajo las condiciones de procesamiento: temperatura, caudal, densidad y características reológicas del mismo.

Debido a que se desea modelar el proceso de extrusión en la parte final del tornillo y en el cabezal, el flujo del material fundido posee bajas velocidades y deformaciones de corte $(\gamma)$, estas últimas son del orden de 50 a 100 1/s, por este motivo se aplica el modelo de Ostwald o Ley de Potencia (Ecuación 2) para el cual se necesitan conocer los valores de $\mathrm{k}$ y $\mathrm{n}$, los cuales se obtienen del ensayo reológico.

$$
\eta=\mathrm{k} \cdot \gamma(n-1)
$$

El valor $\mathrm{n}$ es el exponente de la ley de potencia y se define como la relación entre el esfuerzo y la velocidad de deformación o deformación de corte $(\gamma)$ y el valor $\mathrm{k}$ es la viscosidad a régimen de corte o velocidad de deformación $=0[4]$.

El ensayo reológico se realiza con un reómetro capilar especial conectado a un equipo extrusor [6] y se obtienen los valores de las deformaciones de corte vs viscosidades para 6 puntos o condiciones de medición (Tabla 2).

\subsection{Métodos}

\subsubsection{Dibujo del canal de flujo en el cabezal:}

Se dibuja el canal de flujo en cabezal existente mediante el uso de un software de Diseño Asistido por Computadora en sólido 3D (Figura 1). 


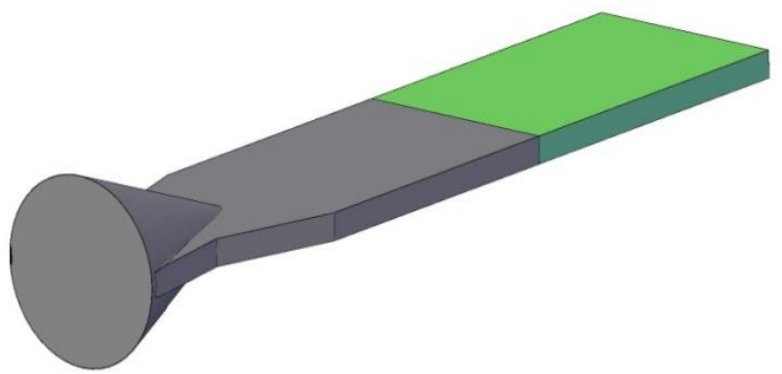

Figura 1: Geometría flujo interior cabezal y salida distancia $60 \mathrm{~mm}$ (verde)

\subsubsection{Simulación}

Se importa el dibujo de la geometría del canal de flujo en el cabezal en el software de simulación ANSYS Polyflow.

Se realiza el mallado de la geometría (Figura 2) utilizando el sistema por defecto del Polyflow, el cual realiza la subdivisión automática en elementos tetraédricos anisotrópicos para flujos en 3 dimensiones que poseen superficies libres como es el que se presenta en nuestro caso (flujo de material fundido a la salida del cabezal).

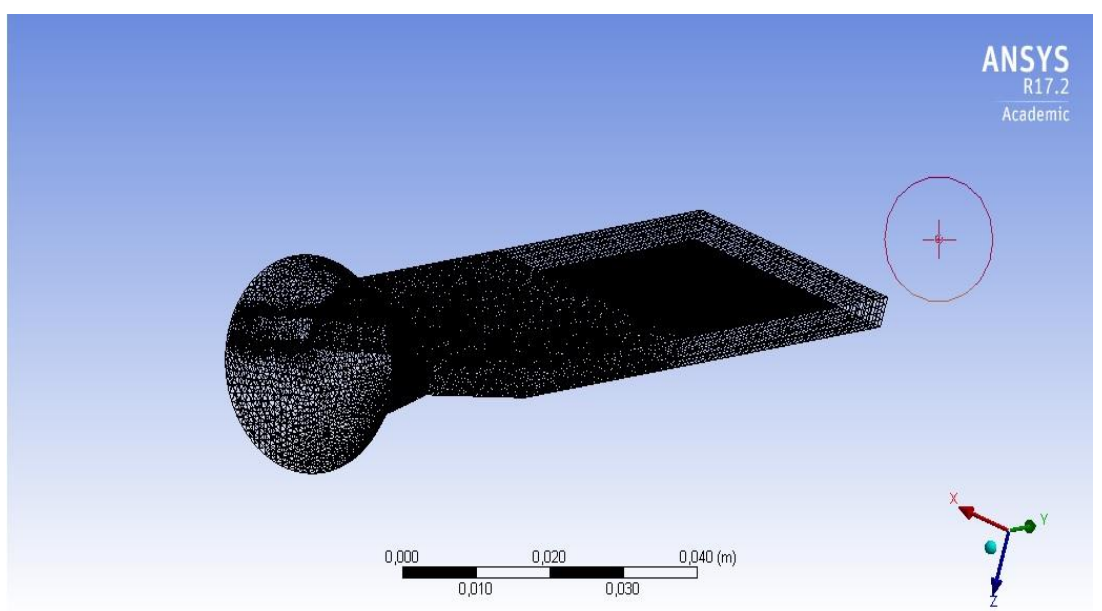

Figura 2: Mallado del canal de flujo en el interior del cabezal.

En el mísmo módulo se le incorporan las denominaciones a cada subdomínio, los cuales son las zonas del fundido dentro del cabezal: SD1(Figura 3) y fuera del mismo: SD2 (Figura 4) para que el software de simulación reconozca cada zona para sus cálculos posteriores. 


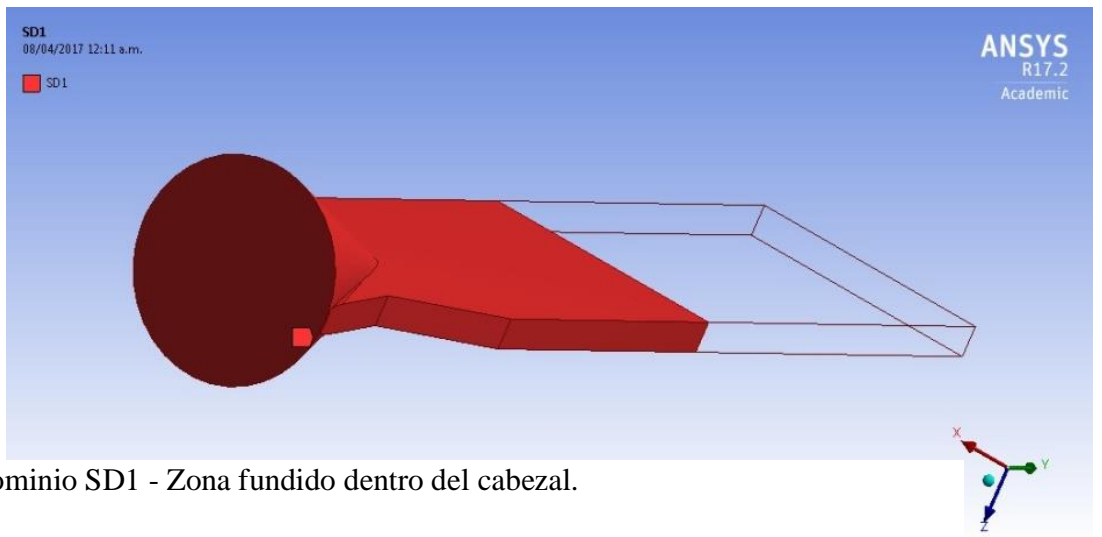

Figura 3: Subdominio SD1 - Zona fundido dentro del cabezal.

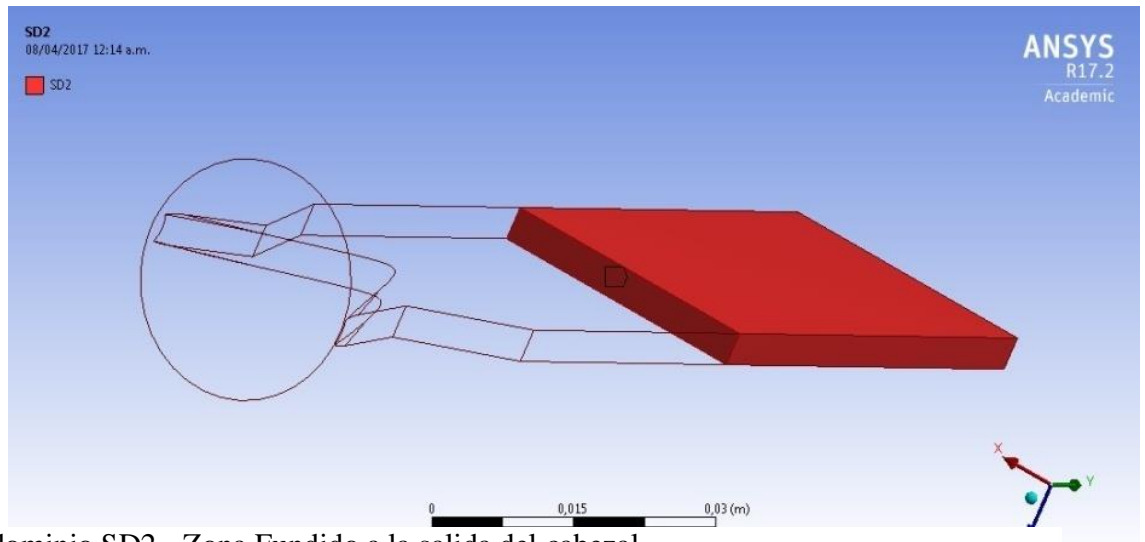

Figura 4: Subdominio SD2 - Zona Fundido a la salida del cabezal.

Se le definen las denominaciones a los contornos (superficies) de cada subdominio SD1_BS2 (Figura 5) y SD2_BS2 (Figura 6) y también a las superficies de entrada del flujo al cabezal: SD1_BS1 (Figura 7) y de salida del flujo del mismo: SD2_BS1 (Figura 8).

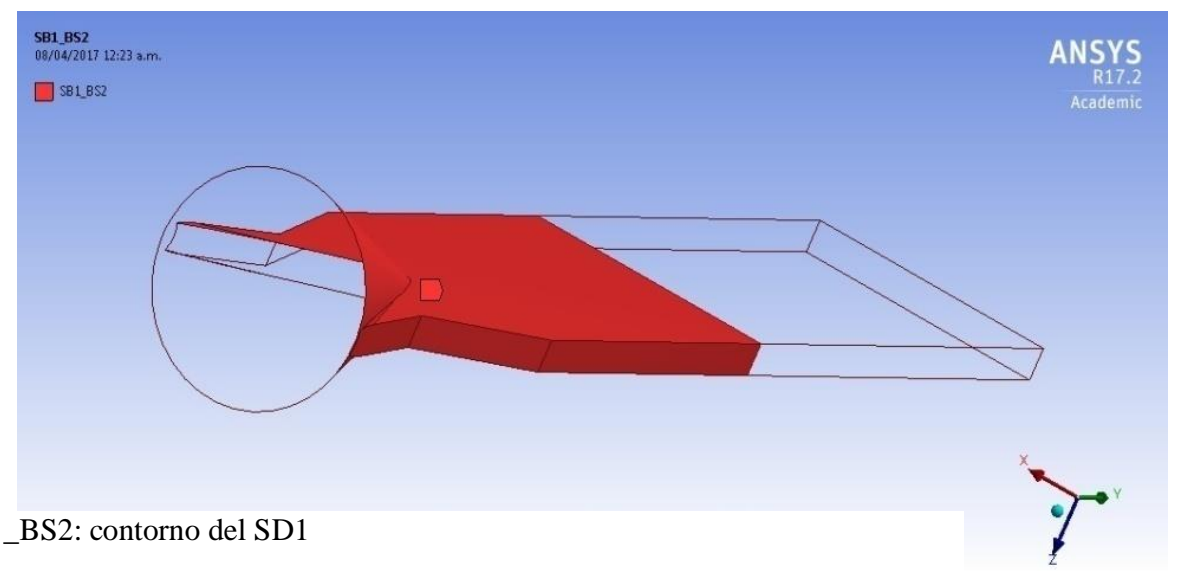

Figura 5: SD1_BS2: contorno del SD1 


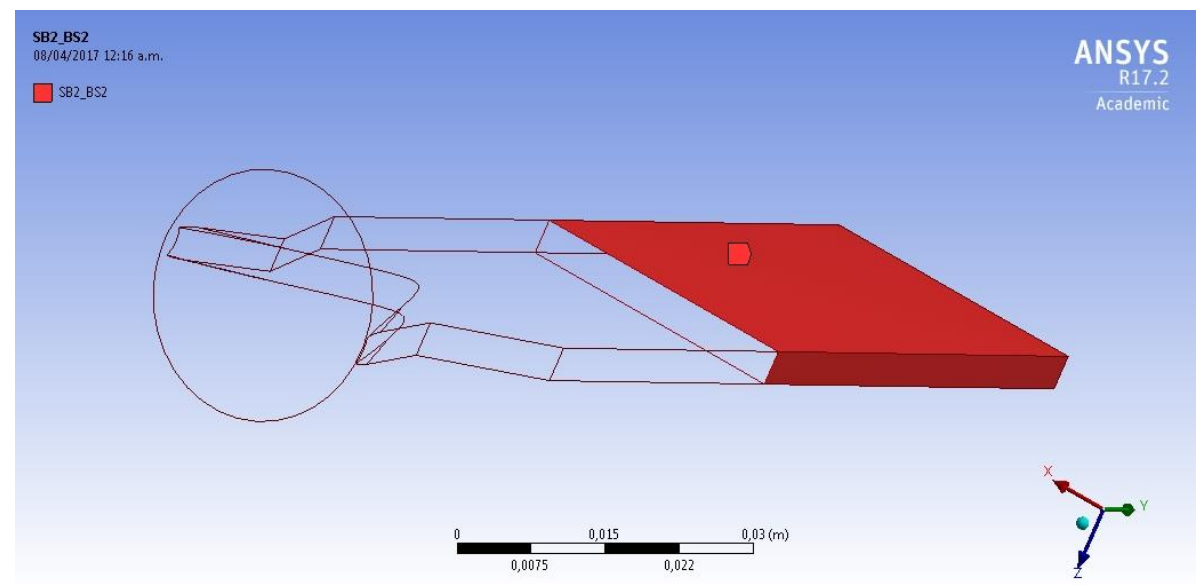

Figura 6: SD2_BS2: contorno del SD2

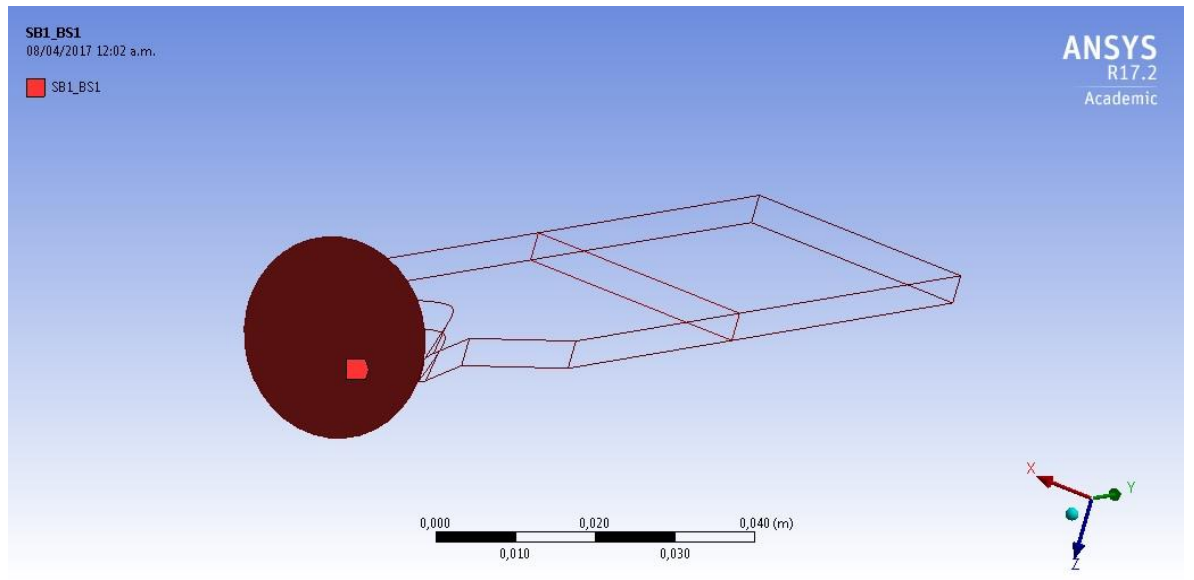

Figura 7: SD1_BS1: superficie entrada flujo al cabezal

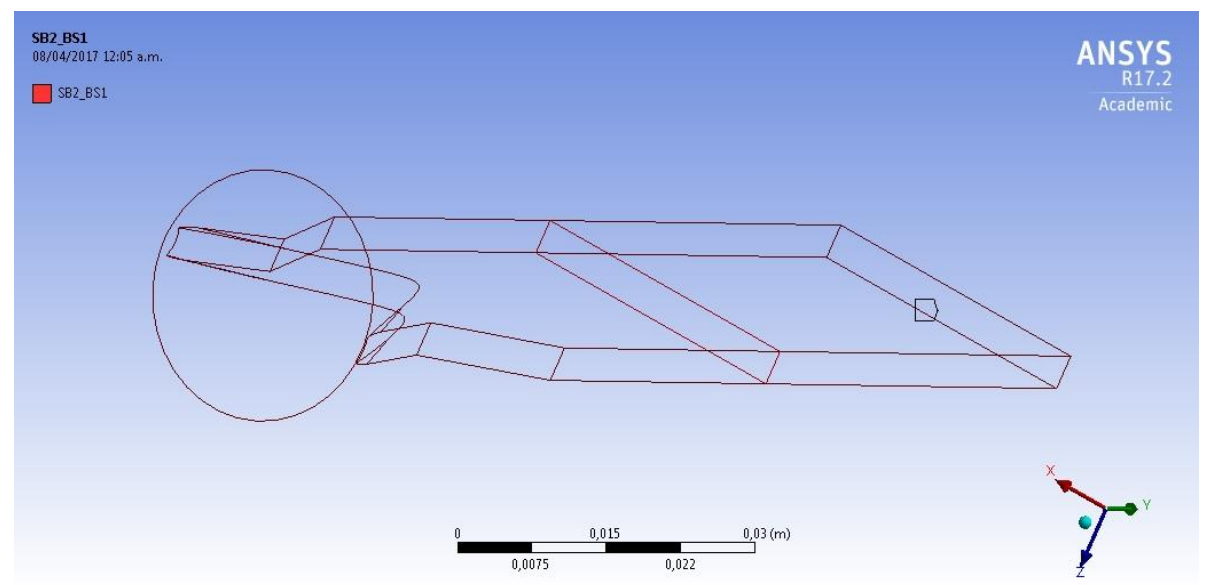

Figura 8: SD2_BS1: Superficie salida flujo del cabezal

Posteriormente se ingresa al módulo Setup y mediante el uso del programa Polydata se introducen las variables de entrada: caudal $(8,5 \mathrm{~kg} / \mathrm{h})$, densidad del material $\left(1,42 \mathrm{~g} / \mathrm{cm}^{3}\right)$ y los valores reológicos $\mathrm{k}=7645 \mathrm{y}$ $\mathrm{n}=0,69$ de la Ley de Potencia, definiéndose también en el mismo las condiciones de borde, es decir los valores de velocidad, tensiones, flujos de entrada y salida, entre otros parámetros de cada subdomínio y contornos (superficies). 
Además, en este paso se define en la programación la zona en la cual debe realizarse nuevamente el mallado y es la que corresponde a la salida del flujo fundido del cabezal (ver región color verde en la Figura 1) para que se obtenga la geometría del perfil en estado fundido con las deformaciones que son producto del efecto de hinchazón del fundido [4].

Este efecto se produce cuando un fluído non-newtoniano (polímero en estado fundido viscoelástico), al atravesar una zona restringida, como es la geometría interna del canal de flujo en el cabezal, se generan en su interior tensiones y al salir de dicha matriz, el material se relaja, disminuyendo su longitud y aumentando su sección transversal.

Para determinar la magnitud de ésta variación geométrica del flujo a la salida del cabezal, ANSYS Polyflow emplea una técnica que se denomina optimización por remallado, la cual utiliza una ecuación cinemática $\mathrm{f}(\mathrm{s})$ que introduce términos no lineales en el problema que conducen a la convergencia del modelo.

Como en nuestro caso tenemos un problema de un flujo numéricamente complejo por ser un fluido non-newtoniano con bajos índices de Ley de Potencia (los valores reológicos se determinaron a velocidades de deformación de entre 50 a 100 1/s) que son los que se pudieron medir con el reómetro capilar especial utilizado en línea con una extrusora [8], se utiliza un esquema numérico incremental que facilita la convergencia.

Para resolver estos problemas altamente no lineales, se calcula primero una solución a bajo caudal y luego se proyecta la misma para un caudal mayor hasta llegar al valor requerido.

Este método se denomina "Evolution" es decir que el caudal evoluciona de un valor inicial, (ver Figura 9), y luego va incrementando el parámetro S y encuentra una segunda solución y así sucesivamente hasta optimizar el modelo, pudiendo existir varios pasos intermedios y en cada uno de ellos se obtiene la solución del paso anterior hasta llegar a la convergencia.

El concepto puede aplicarse a distintas condiciones de contorno (caudal, temperatura, fuerza de arrastre, cantidad de deslizamiento de masa, etc.) y propiedades del material (índice de adelgazamiento por cizallamiento, tiempo de relajación, calor específico, etc.)

Este proceso se realiza automáticamente y los incrementos se adaptan de la misma forma, hasta encontrar la mejor solución. A continuación se define el significado de cada uno de los términos de la ecuación aplica éste método y los valores de cada uno de ellos, mostrando en la Figura 9 como es la evolución del parámetro $\mathrm{S}$ :

$\mathrm{Q}=$ Qnom. . $\mathrm{f}(\mathrm{s})$

Qnom. es el valor del caudal nominal= 8,5 kg/h.

$\mathrm{f}(\mathrm{s})$ es la función evolución.

"s" es la variable de la evolución.

Sinicial $=1,33=1 / \mathrm{n}$ ( siendo $\mathrm{n}=0,69$, el valor del exponente de la Ley de Potencia y que se obtuvo del ensayo reológico).

Sfinal $=1,44=1 / \mathrm{n}$ ( siendo $\mathrm{n}=0,75$, el valor mínimo de $\mathrm{n}$ que asegura la linealidad y convergencia del modelo).

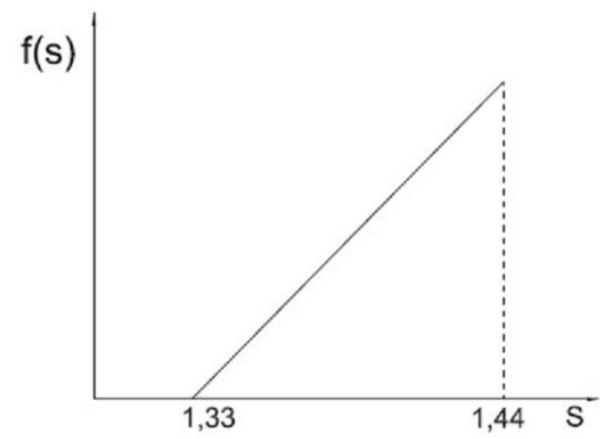

Figura 9: Evolución del parámetro $S$

A continuación, en Solución se hace la corrida computacional para calcular los resultados para el modelo definido en los pasos anteriores. 
Los valores de la distribución de velocidades, deformaciones de corte, presiones, contornos del perfil a la salida del cabezal y las gráficas de cada uno de ellos para su análisis se obtienen mediante el Posprocesado en la última fase denominada Results. .

\subsubsection{Diseño Experimental y Construcción del Calibrador:}

Se diseña experimentalmente el calibrador y se dibuja con un software de diseño asistido por computadora en sólido 3D (Figura 10).

Se construyen las distintas partes que componen el calibrador y se las ensambla (Figura 11), ubicando el conjunto en el recinto o batea de calibración-enfriamiento y dejando los tornillos de fijación de la parte superior sin ajustar completamente, dejando el juego necesario para introducir el perfil para su calibración.

El calibrador define las dimensiones externas finales del perfil, [1], de manera que el perfil en estado fundido a la salida del cabezal, a temperaturas cercanas a los $200^{\circ} \mathrm{C}$, entra al calibrador, donde se aplica vacío y el material copia las dimensiones internas del calibrador.

$\mathrm{Al}$ estar inmerso el calibrador en una batea con agua a $20^{\circ} \mathrm{C}$, el perfil se enfría, solidificándose, por lo cual para definir las dimensiones internas del calibrador se tuvo en cuenta que el material fundido al enfriarse, se contrae y en el caso de los compuestos de PVC rígido la misma puede llegar a valores elevados de hasta un $4 \%$.

Se debe tener en cuenta también que se genera otra disminución del área transversal del perfil todavía en estado viscoelástico que le provoca el estiramiento realizado por el sistema de arrastre para que pueda avanzar el perfil y que puede ser de entre el 5 al $10 \%$ para perfiles de PVC rígido de bajo espesor [6].

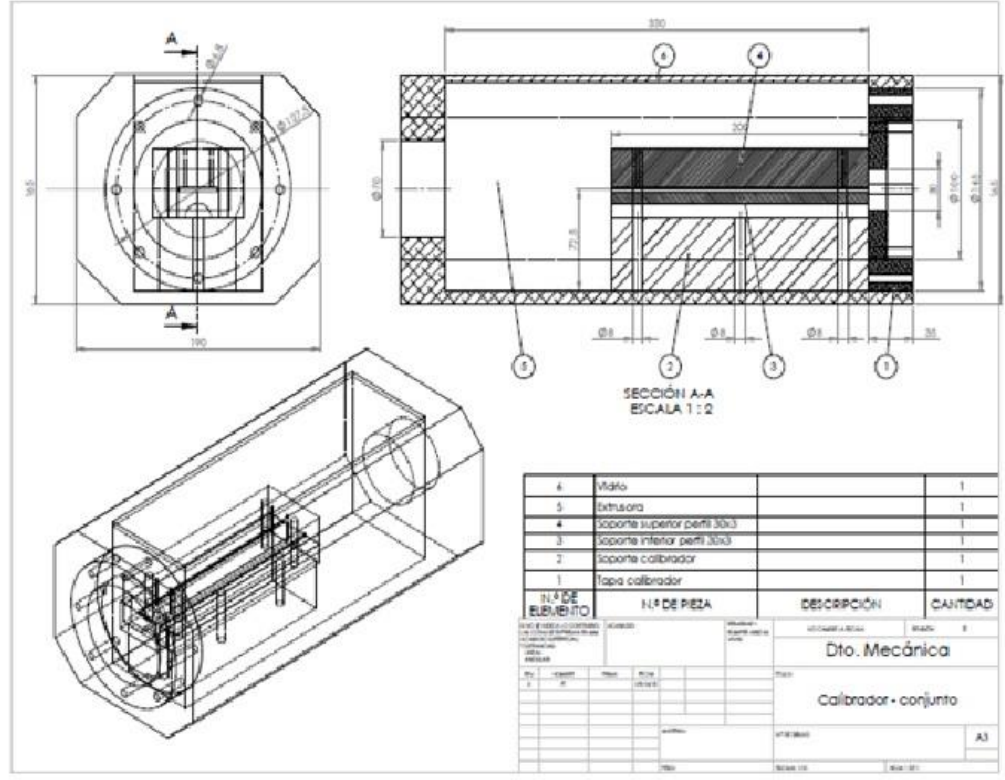

Figura 10: Plano del calibrador CAD-3D

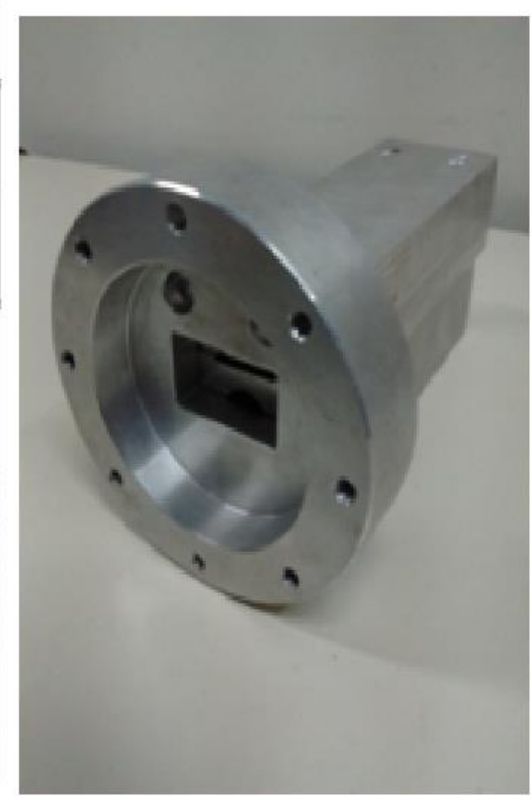

Figura 11: Calibrador ensamblado

\subsubsection{Pruebas de Extrusión}

Se realizan pruebas de extrusión utilizando una extrusora monotornillo Collins (Figura 12) con un tornillo de relación de compresión 2:1, el equipamiento auxiliar de la misma marca, como ser las bateas de calibración por vacío y enfriamiento (Figura 13) y el sistema de arrastre o tiro(Figura 14). 


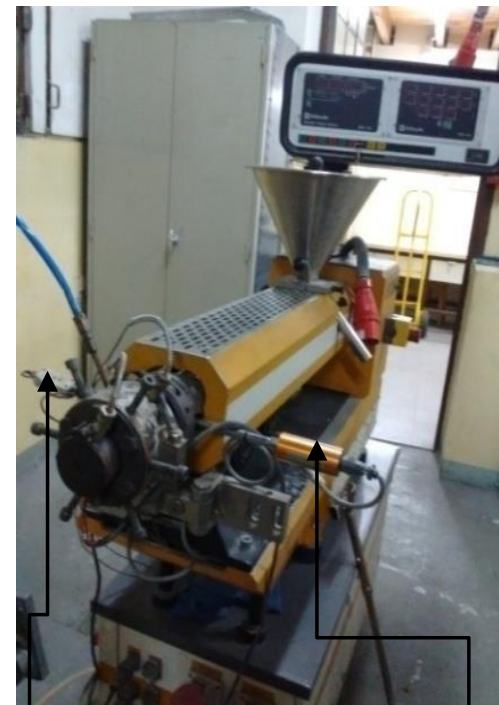

Figura 12: Extrusora Collins

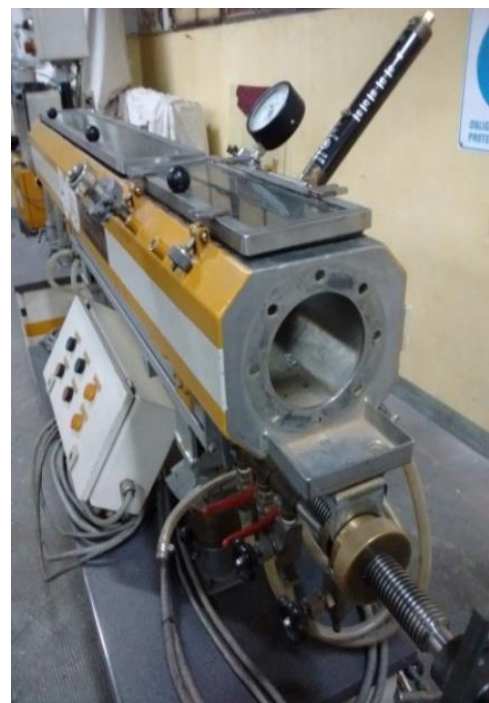

Figura 13: Batea de calibración por vacío y enfriamiento

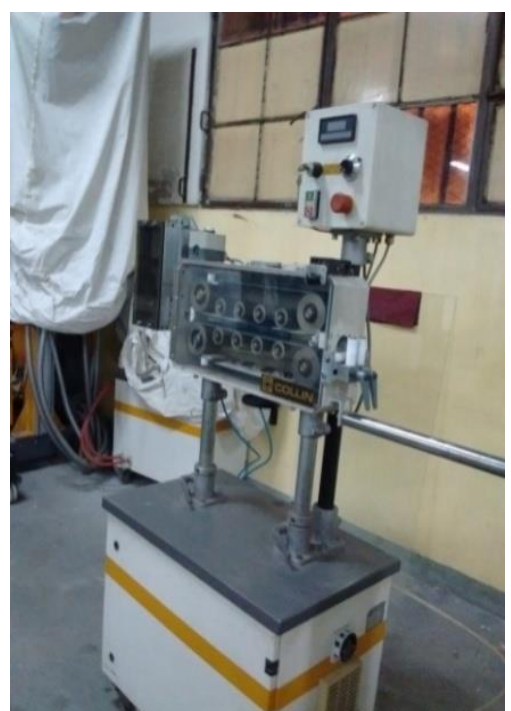

Figura 14: Unidad de arrastre

Sensor de Temperatura

Sensor de presión

\section{RESULTADOS}

\subsection{Valores Reológicos}

Los valores reológicos se obtuvieron del ensayo realizado por personal técnico de la empresa ALFAVINIL S.A., fabricantes de compuestos de PVC, utilizando un reómetro capilar RheoDrive 7, modelo: Rheomex 19/25 OS, en línea con una extrusora, cuyo tornillo posee una relación de compresión 2:1, L/D: 25 y una matriz capilar de hendidura $\mathrm{H}: 2,0 \mathrm{~mm}$ W: $20 \mathrm{~mm}$ y con un perfil de temperaturas del material de $150^{\circ} \mathrm{C}$, $170^{\circ} \mathrm{C}$ y $190^{\circ} \mathrm{C}$ en la extrusora y $200^{\circ} \mathrm{C}$ en el cabezal.

Tabla 2: Valores reológicos obtenidos del ensayo del PVC rígido - ALFAVINIL código: RE97/1-773

\begin{tabular}{l|l|l|l|l|l}
\hline $\begin{array}{l}\text { PUNTOS } \\
\text { DE MEDI- } \\
\text { CIÓN }\end{array}$ & $\begin{array}{l}\text { DEFOMACIÓN } \\
\text { DE CORTE ( }) \\
1 / \mathrm{s}\end{array}$ & $\begin{array}{l}\text { ESFUERZO } \\
\text { DE CORTE (T) } \\
\text { Bar }\end{array}$ & $\begin{array}{l}\text { VISCOSIDAD } \\
(\eta) \\
\mathrm{Pa}\end{array}$ & $\begin{array}{l}\text { CAUDAL } \\
\text { MÁSICO (Qm) } \\
\mathrm{g} / \mathrm{h}\end{array}$ & $\begin{array}{l}\text { CAUDAL VOLU- } \\
\text { MÉTRICO (Qv) } \\
\mathrm{cm}^{3} / \mathrm{s}\end{array}$ \\
\hline 1 & 22,7 & 0,7 & 2933,7 & 1730,4 & 0,302 \\
\hline 2 & 27,5 & 0,8 & 2725,4 & 2101,2 & 0,367 \\
\hline 3 & 38,6 & 1,0 & 2511,2 & 2944,4 & 0,514 \\
\hline 4 & 51,6 & 1,2 & 2329,8 & 3940,8 & 0,688 \\
\hline 5 & 69,8 & 1,4 & 2069,3 & 5324,8 & 0,930 \\
\hline 6 & 90,4 & 1,7 & 1881,5 & 6899,2 & 1,205 \\
\hline
\end{tabular}

\subsection{Simulación}

3.2.1 Distribución de la Velocidad en Diferentes Secciones: 
En la etapa de Posprocesado de la simulación se definen secciones transversales a determinadas distancias en el canal de flujo, para analizar la distribución de los vectores de velocidad en una misma sección y en cada una de ellas (Figura 15).

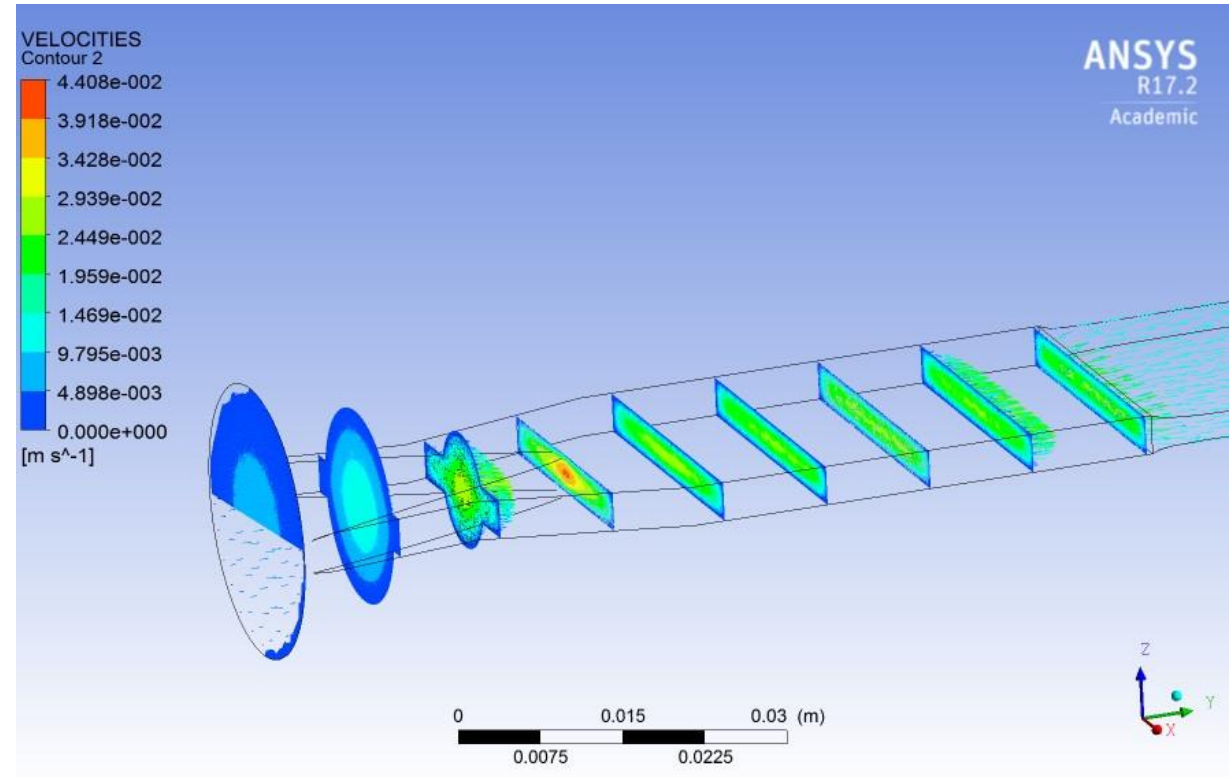

Figura 15: Distribución de velocidades dentro y a la salida del cabezal

Se puede observar que en las regiones centrales de las secciones en la zona de alimentación (zona cónica), estos vectores de velocidad poseen mayor valor (zona celeste) que en los contornos ya que como el flujo del material se encuentra cercano a la pared interior del canal de flujo, la velocidad es cero (zona azul), esto es coherente con lo que ocurre en la realidad cuando un fluido circula por un conducto, el perfil de velocidades será máximo en el centro y tendiente a cero en regiones cercanas a las paredes interiores del mismo.

A medida que avanza el flujo del material fundido se pueden observar vectores de velocidad en las zonas centrales de cada sección transversal de mayor magnitud (color verde más intenso), esto es debido al cambio abrupto de la geometría en el canal de flujo pasando de cónica a rectangular, en particular si analizamos la cuarta sección transversal desde la entrada del flujo al cabezal, se observa que en el centro hay una zona de vectores de velocidad aún de mayores magnitudes (color rojo).

Las variaciones en los vectores de velocidad en cada punto de una misma sección ocasiona que el canal de flujo no cumpla con el principio de diseño de "mínimo volumen" de los cabezales, es decir que el fundido debe llegar al mismo tiempo a todos los puntos de la sección transversal o con la misma velocidad

Estas diferencias entre el deslizamiento del fluido en las zonas centrales con respecto a las que están más cercanas a la pared interior del cabezal originan tensiones internas por cizallamiento en el material fundido que posteriormente podrían conducir a la aparición de fallas en servicio; esto se hace más crítico en perfiles de formas complejas y en el caso particular que entre el ancho y el espesor exista una importante diferencia dimensional.

\subsubsection{Distribución de Deformaciones de Corte}

En el gráfico que se muestra a continuación (Figura 16) se observa que en la zona donde cambia la geometría se generan deformaciones de corte (muy elevados todos los colores menos el azul), esto nos indica que en dichos lugares, el material fundido puede llegar a degradarse, en especial para nuestro caso en el que se usa PVC rígido, por la generación de elevadas temperaturas localizadas por fricción y cizallamiento.

Es aconsejable, para poder disminuir o evitar que se genere este efecto perjudicial, modificar la geometría del canal de flujo del cabezal para que la transición entre ambas zonas sea suave. 
Los resultados de la simulación predicen en este caso los efectos perjudiciales que se producen con el actual diseño de cabezal, de manera que si es necesario construir un nuevo cabezal, se debe modificar la geometría, de tal manera que las deformaciones de corte sean bajas y en lo posible que sus valores se encuentren entre $50 \mathrm{a} 100 \mathrm{1} / \mathrm{s}$, que es lo aconsejable para procesar este tipo de material y como se describía en el ensayo reológico, son los valores donde tiene validez la Ley de Potencia o de Ostwald.

Otro análisis que se realiza es sobre las zonas del contorno a la entrada y a la salida del material fundido del cabezal, en las cuales las deformación de corte es cero, en especial si se deja una zona paralela final dela matriz suficiente para tener un hinchazón del fundido mínimo y dentro de los parámetros que se recomienda [7].

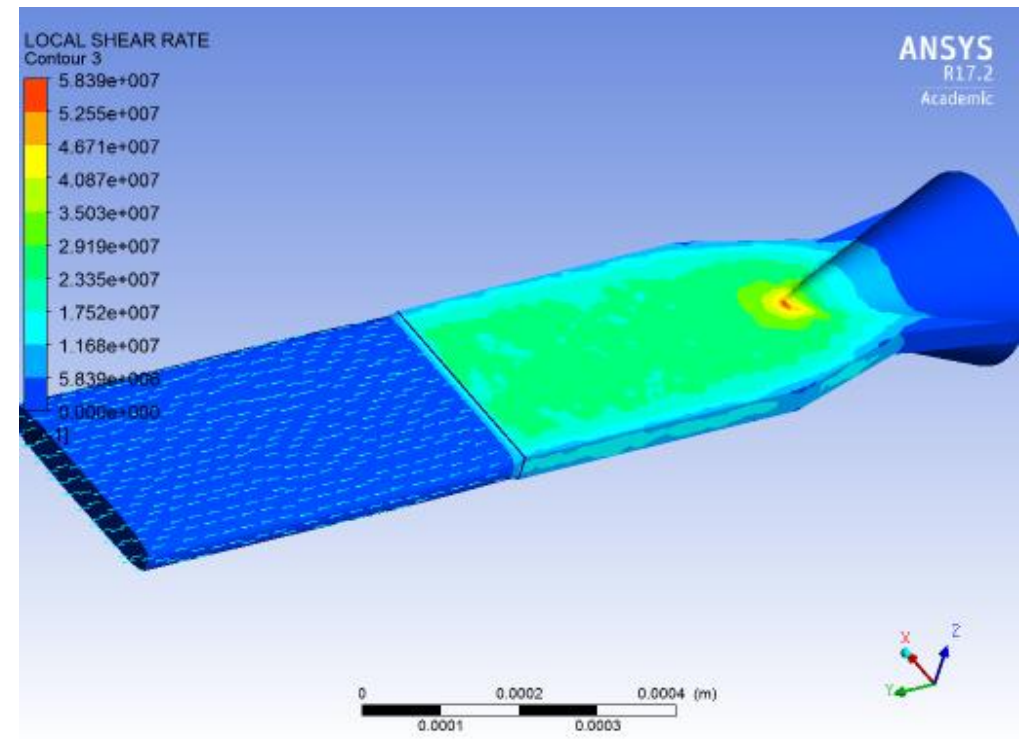

Figura 16: Distribución de Deformaciones de Corte

\subsubsection{Comparación contornos perfil original vs perfil fundido salida cabezal}

Otro de los resultados importantes que se obtienen de la simulación es el contorno geométrico del perfil en estado fundido a la salida del cabezal a una distancia de $60 \mathrm{~mm}$.

Para ello se modifica la orientación de los ejes de la geometría del canal en la etapa de Posprocesado, eliminando todos los contornos y superficies a excepción de los correspondientes al perfil requerido a la salida del cabezal y al del flujo del material fundido a la salida del cabezal a una distancia de $60 \mathrm{~mm}$.

Al superponerse ambos contornos se pueden apreciar las diferencias geométricas y dimensionales que existen entre ambos; a continuación se exportan estas geometrías y se dimensionan las diferencias (Figura 17).

Las mismas se deben a lo comentado anteriormente de la incidencia del efecto de hinchazón del fundido que provoca un aumento del área transversal del flujo del material fundido a la salida del cabezal.

En una simulación anterior, se utiliza un modelo de geometría del canal con una zona a la salida del cabezal de $10 \mathrm{~mm}$, con la cual las deformaciones fueron mayores, existiendo importantes diferencias geométricas y dimensionales, lo que hubiera ocasionado que el perfil no se hubiera podido calibrar y tampoco fabricar, porque se hubiera atascado en el calibrador.

Optimizándose así el diseño del modelo al aumentar la longitud de la zona a salida del perfil fundido con una nueva simulación a una distancia de $60 \mathrm{~mm}$.

La variación dimensional a una distancia de $60 \mathrm{~mm}$ del cabezal se puede apreciar en la Tabla 3:

Tabla 3: Variaciones dimensionales entre el perfil original y de salida del cabezal a una distancia de $60 \mathrm{~mm}$.

\begin{tabular}{l|c|c|c}
\hline \multicolumn{1}{c|}{ CARACTERÍSTICAS } & PERFIL ORIGINAL & \multicolumn{2}{|c}{ PERFIL A LA SALIDA CABEZAL } \\
\hline & $\mathrm{mm}$ & $\mathrm{mm}$ & DIFERENCIA DIMENSIONAL (\%) \\
\hline Espesor & 3,0 & 3,08 & 2,7 \\
\hline
\end{tabular}




\begin{tabular}{l|l|l|l}
\hline Ancho & 30,0 & 31,8 & 6,0 \\
\hline
\end{tabular}

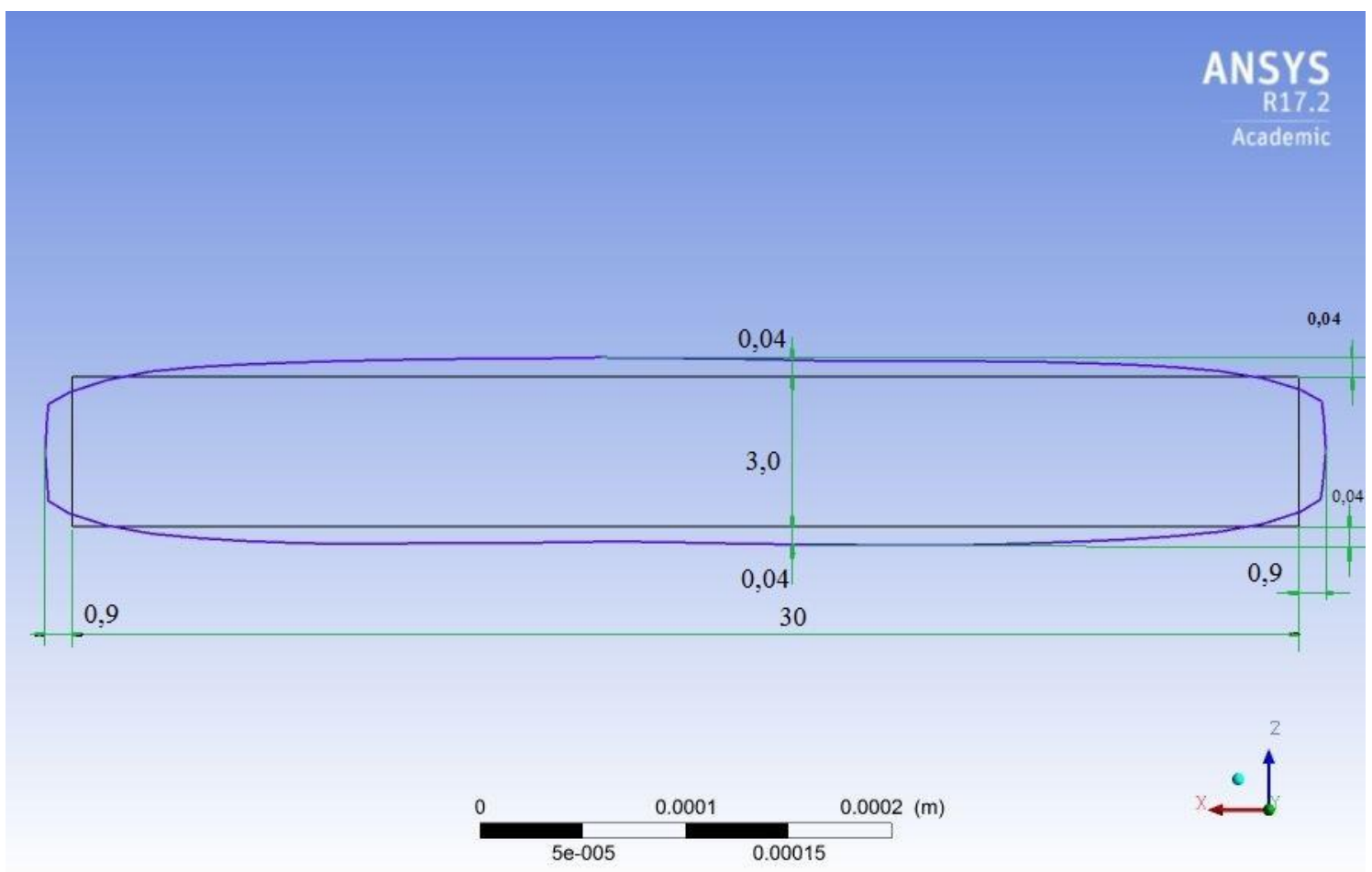

Figura 17: Comparación contornos perfil original (negro) y a la salida del cabezal (azul)

Por lo tanto se lograron valores máximos de deformación por el efecto de hinchazón del fundido dentro de los parámetros recomendados, los cuales son de 3 a $6 \%$ para espesores de perfiles de PVC rígido entre 3 a $4 \mathrm{~mm}$ de espesor [7].

\subsubsection{Distribución de presiones:}

Se obtuvo el gráfico con la distribución de presiones, en el cual se observa que a la entrada del flujo del material fundido al cabezal la presión es máxima y es la posee el material fundido en la extrusora a la salida de la zona de dosificación, con valores que dependen del caudal (revoluciones por minuto del tornillo) y de la temperatura del material, como así también del diseño del tornillo y matriz (contrapresión).

La presión disminuye al avanzar el flujo dentro del cabezal, como se puede observar en la figura 18, de manera que a la salida de la matriz (zona de color azul), la misma es cero ya que el flujo del material fundido está libre de tensiones.

Se observa que el valor de presión obtenido de la simulación en la zona donde se encuentra el sensor de presión en el cabezal (color celeste) posee valores entre 142 y 68 Bar(en la figura 19 las presiones están expresadas en Pascal $(\mathrm{Pa})$ y el valor medido con dicho sensor de presión es de 51 bar para un caudal de 8,5 $\mathrm{kg} / \mathrm{h}$ y una temperatura medida con otro sensor en el cabezal de $200^{\circ} \mathrm{C}$.

Esta diferencia entre las presiones simuladas y reales del proceso puede ser debida a las elevadas deformaciones de corte que se producen en el interior del cabezal y que al utilizar el modelo de la Ley de Potencia o de Ostwald, el mismo es para deformaciones mucho más bajas y del orden de entre 50 a 100 1/s.

La gran diferencia entre las deformaciones de corte obtenidas como resultado de la simulación (entre $5,210^{7}$ a $5,610^{7} 1 / \mathrm{s}$ en los valores más elevados de color rojo) y la que utiliza la Ley de Potencia afectan los resultados de la distribución de presiones que entrega el software. 


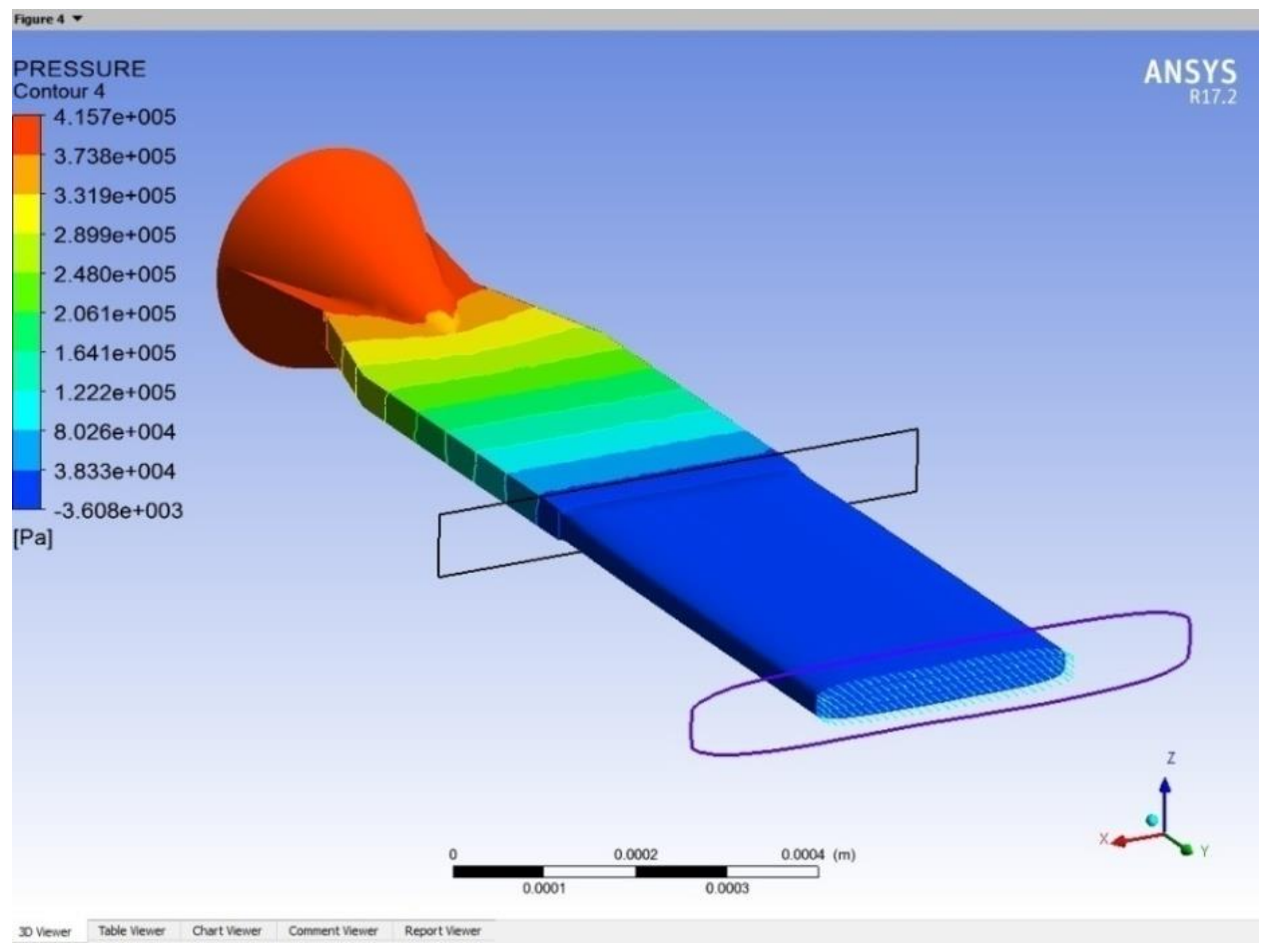

Figura 18: Distribución de presiones dentro y fuera del cabezal

\subsection{Pruebas con el equipo extrusor}

Se realiza una optimización del proceso y del diseño del calibrador mediante tres pruebas realizadas con la extrusora, sus equipos auxiliares y el calibrador experimental instalado.

La conclusión de estos ensayos en máquina mostró que se deben realizar pequeñas modificaciones en el diseño del calibrador, como ser ranuras para mejorar la aplicación del vacío y además se ajustaron los parámetros de máquina, en particular las revoluciones del tornillo y la velocidad de arrastre.

Dichas pruebas se realizan a una temperatura del material fundido en el cabezal de $200^{\circ} \mathrm{C}$, obtenida mediante un sensor ubicado en el mismo (Figura 12) y modificando el caudal por medio de la variación de las revoluciones del tornillo de la extrusora.

Se registranlos valores de la presión en el cabezal mediante otro sensor (figura 13) que posee el equipo y que también está ubicado en el cabezal, observando su valor en el panel de control de la extrusora.

A continuación se detallan cada una de estas pruebas:

\subsubsection{Primer prueba}

Se ajusta la velocidad del tornillo para un caudal másico de $5 \mathrm{Kg} / \mathrm{h}$, midiendo con el sensor una presión $=55$ bar; siendo las dimensiones del perfil extrudado a la salida del calibrador de ancho $=26 \mathrm{~mm}$ y espesores: 2,6 $\mathrm{mm}$ en un lado y $2,9 \mathrm{~mm}$ en el otro lado del ancho.

Se determina que la causa de no llegar alas dimensiones requeridas $($ ancho $=30 \mathrm{~mm}$ y espesor $=3 \mathrm{~mm}$ ) es un déficit de vacío en la batea calibración por vacío y enfriamiento (Figura 14). 
El vacío es generado por una bomba que se encuentra en el depósito de agua que está ubicado en la parte inferior de la batea de calibración por vacío y enfriamiento y éste vacío es medido con un instrumento de medición de presión o manómetro (Figura 19).

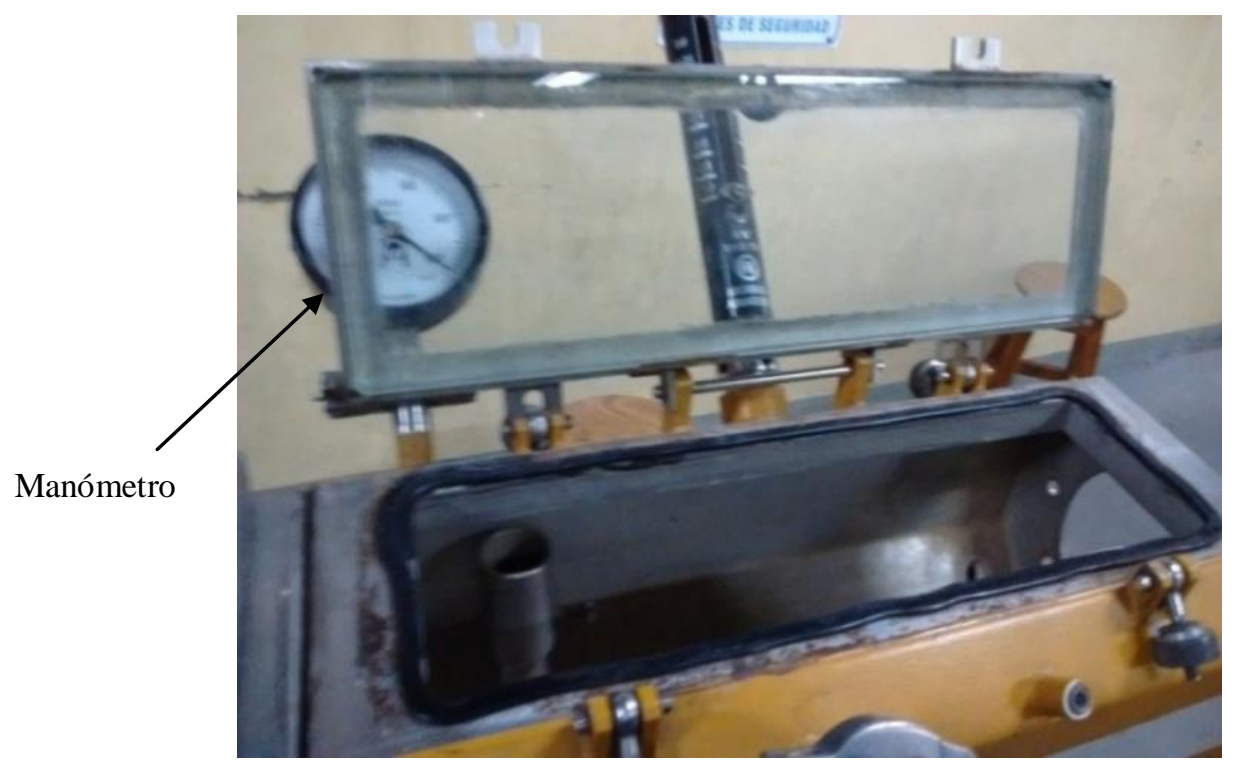

Figura 19: Batea de calibración por vacío y enfriamiento

\subsubsection{Segundaprueba}

Se ajustaron las revoluciones del tornillo a $48 \mathrm{rpm}$ de manera de obtener un caudal másico de $5,7 \mathrm{~kg} / \mathrm{h}$ y se midió una presión de 47 Bar.

Para aumentar el vacío se le realizaronal calibrador una ranuras internas que comunican el lugar (hueco) donde está definida la geometría final del perfil, con el recinto donde se aplica el mencionado vacío.

Se midieron las dimensiones del perfil a la salida del calibrador y se obtuvieron los siguientes valores: ancho $=29,6 \mathrm{~mm}$ y espesores en la zona central del perfil $=3 \mathrm{~mm}$ y en cada extremo del ancho= $2,7 \mathrm{~mm}$.

Por lo cual, si bien se mejora el valor dimensional del ancho, en los lados todavía no se llega a copiar bien la geometría y además la geometría no es rectangular, ya que el espesor en la parte central es de $3 \mathrm{~mm}$ y disminuye hacia los bordes llegando a un valor en ambos extremos de 2,7 mm, por lo cual, el proceso no es apto aún para corregir este defecto.

\subsubsection{Tercer prueba}

Se modifica nuevamente las revoluciones del tornillo a $65 \mathrm{rpm}$, obteniendo un caudal másico= 8,5 kg/h, es de destacar que este valor de caudal es el mismo que se utiliza como una de las variables de entrada en el software de simulación.

La presión medida fue de 51 bar(en estecaso hay una diferencia con los valores obtenidos en la simulación, ya que para la zona donde está ubicado el sensor de presión, los resultados fueron entre 142 y 68 bar.

El incremento del caudal másico, acompañado por un aumento de la velocidad de arraste y utilizando el calibrador con las ranuras para mejorar la aplicación del vacío sobre el flujo del material fundido que proviene del cabezal y que se está calibrando y enfriando, logra que se obtenga la geometría deseada del perfila la salida del calibrador. 
Por otra parte, se logran las dimensiones requeridas con un error menor al $5 \%$ en los extremos del ancho; ya que se midió con un pie coliza que el ancho $=30 \mathrm{~mm}$ y en los extremos $=2,9 \mathrm{~mm}$, es decir elerror fue del $3,3 \%$.

La medición del caudal fue realizada tomando la cantidad de material que se extruda por unidad de tiempo y el mismo coincide con el caudal que se utilizó como variable de entrada en el software de simulación, es decir $8,5 \mathrm{~kg} / \mathrm{h}$, trabajando el tornillo a $65 \mathrm{rpm}$.

La presión censada fue de 51 bar y la que se obtuvo del software de simulación osciló entre 142 y 68 bar y la explicación de éstas diferencias se deben a que el modelo utilizado de Ley de Potencia es para bajas velocidades de deformación y en la simulación dichas deformaciones de corte fueron muy elevadas, en especial en la zona donde se modificaba la geometría del canal de cónica a rectangular (zona de transición).

\section{DISCUSIÓN}

El resultado de la primer simulación utilizando una geometría del canal de flujo con una zona a la salida del cabezal de $10 \mathrm{~mm}$ (Figura 20 zona de color verde) generaba elevadas deformaciones por el efecto de hinchazón del fundido, lo cual no hubiera sido posible calibrar y fabricar el perfil a las dimensiones requeridas, como se explicaba anteriormente.

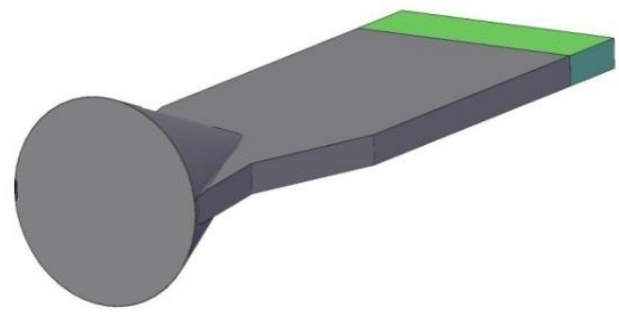

Figura 20: Geometría canal flujo zona salida de $10 \mathrm{~mm}$ (zona color verde).

Por lo tanto, se modifica el modelo aumentando la longitud de dicha zona a $60 \mathrm{~mm}$ y se simula nuevamente obteniendo valores de deformación que se encuentran dentro de lo recomendado.

Esta longitud de la zona del perfil fundido fuera del cabezal es la que se aconseja utilizar para la extrusión del mismo, es decir la distancia entre el cabezal y el calibrador debería ser de dicha magnitud para asegurarnos una calibración adecuada y no tener atascamientos cuando el perfil en estado fundido ingresa al calibrador.

Al aplicarse vacío en la batea de calibración-enfriamiento, el material fundido en el calibrador aumenta su sección transversal copiando las dimensiones internas del mismo y avanzando con un flujo continuo por medio de la fuerza de arrastre, mientras se solidifica al estar inmerso en agua a $20^{\circ} \mathrm{C}$.

Además el software utiliza la optimización por remallado en dicha zona de salida y que es la que se muestra en este trabajo, ya que al aumentar la longitud de la zona de flujo libre a la salida del cabezal, la deformación por el efecto de hinchazón del fundido disminuye.

\section{CONCLUSIONES}

Mediante la simulación se obtiene un modelo de geometría del perfil a la salida del cabezal de 60 mm de longitud logrando de esta forma minimizar el hinchazón del fundido y obtener una deformación con diferencias dimensionales que se encuentran dentro de los porcentajes recomendados, como se describe en el punto 3.2.3.

La longitud obtenida de $60 \mathrm{~mm}$ de la simulación es la recomendada a utilizar en el proceso de extrusión para la puesta a punto de la distancia que debe existir entre el cabezal y el calibrador con el fin de evitar problemas de atascamiento del material fundido que ingresa al calibrador y aplicarse vacío.

De los resultados de la simulación se determina que la distribución de los vectores de velocidad en cada una de las secciones transversales no son homogéneas y también las deformaciones de corte tuvieron 
valores muy elevados, lo que ocasiona que se generen tensiones internas en el perfil que provoquen su falla en servicio.

Por lo cual se debería rediseñar la geometría del canal de flujo del cabezal para lograr mejorar estos parámetros que influyen en las propiedades finales del perfil, como ser la generación de elevadas tensiones internas que afectan la calidad del producto.

Se puede también definir una zona paralela óptima, debido a que su longitud influye en el efecto de hinchazón el fundido, es decir al aumentar la misma se obtiene una mayor relajación de tensiones internas y por consiguiente una menor deformación del material a la salida del calibrador [8].

Se pudo realizar el ensayo reológico y obtener los valores k y n de la Ley de Potencia para el compuesto de PVC rígido del presente trabajo utilizando un reómetro capilar especial conectado en línea con una extrusora y los mismos ser utilizados en un software de simulación que usa Dinámica de Fluidos Computacional y el método de Extrusión Directa, [9] para optimizar el diseño de un cabezal de un perfil rectangular en PVC rígido.

Por lo cual se comprueba la capacidad predictiva del software de simulación ANSYS Polyflow para este trabajo y con ello poder aplicarlo para la simulación de geometrías de perfiles más complejas.

Por último, se diseña y construye un calibrador específico para este perfil y en sólo tres pruebas con el equipo extrusor se optimiza el funcionamiento del mismo y del proceso, verificando que el caudal utilizado en la simulación es el correcto, logrando obtener un perfil con la geometría y dimensiones finales requeridas y con un error dimensión al menor al $5 \%$.

\section{AGRADECIMENTOS}

Se agradece especialmente alTécnico Universitario Gustavo Pierson, alIng. Federico Tirapelli y al Técnico Javier Ríos Zabala, personal del Instituto Politécnico Superior-U.N.R. por su colaboración en el diseño, construcción del calibrador y participación en el proceso de extrusión.

A la Dra Patricia Frontini del INTEMA, UndMPy al Dr. Javier Signorelli del IFIR, CONICET Rosario por sus aportes y sugerencias.

Por último al Dr. Fernando Angiolini y al Lic. Leonardo Tort, personal técnico y directivo de la empresa ALFAVINIL S.A., quienes en forma desinteresada prestaron su colaboración para la realización delos ensayos reológicos y aportaron el material para poder realizar las pruebas de extrusión.

\section{BIBLIOGRAFÍA}

[1] SZARVASY, I., SIENZ, J., PITTMAN, J., et al., Computer Aided Optimization of Profile Extrusion Dies, In: International Polymer Processing, XV, 2000.

[2] ZHAO, W., Finit Element Simulation of 3D unsteady viscoelastic free surface flow with level set method, ANTEC, Society of Plastics Engineers Annual, 2010.

[3] SRINIVASAR.et al., Extrusion simulation and experimental validation to optimize precision die design, ANTEC, 2004.

[4] VLACHOPOUlOS, J., FATTAMANN, G., The Role of Rheology in Polymer Extrusion, Department of Chemical Engineering, McMaster University, 2003.

[5] TITOW, M.V., Technology of PVC, 4 ed., Elsevier Applied Science Publishers, 2002.

[6] LIMPER, A., FATTMANN, G., Application of on online rheometer to evaluate the melt properties of $P V C$, Institut für Kunststofftechnik, University of Paderborn, Germany, 2002.

[7] MICHAELI, W. Extrusion Dies for Plastic and Rubber, 3 ed., Munich, Germany, Hanser Publishers, 2003.

[8] GONCALVES, N.D. et al, "Design of complex profile extrusion dies through numerical modeling", Journal of Non-Newtonian Fluid Mechanics, v. 200, pp. 103-110, Oct. 2013.

[9] ANSYS, Polyflow Tutorial Guide, ANSYS, Inc. Release 17.0, Southpointe, USA, January 2016. 